\title{
THE PSEUDOINVERSE OF AN $r$-CIRCULANT MATRIX
}

\author{
W. T. STALLINGS AND T. L. BOULLION
}

\begin{abstract}
It is shown that the Moore-Penrose pseudoinverse $C^{+}$of an $r$-circulant matrix $C$ is always the conjugate transpose of an $r$-circulant matrix. In addition, necessary and sufficient conditions are given for $C^{+}$to be an $s$-circulant matrix. Finally, a method for calculating $C^{+}$is given.
\end{abstract}

I. Introduction. The Moore-Penrose pseudoinverse of a nonsingular $n \times n r$-circulant matrix is an $s$-circulant matrix where $r s \equiv 1(\bmod n)$. A similar statement is valid for some, but not all, singular $r$-circulant matrices. In this paper we show that the pseudoinverse of an $r$-circulant matrix is always the conjugate transpose of an $r$-circulant matrix, and use this result to describe the class of $r$-circulant matrices whose pseudoinverses are $s$-circulant matrices for some integer $s$.

II. Background information.

Definition 1. An $r$-circulant matrix is an $n \times n$ complex matrix of the form

$$
C=\left[\begin{array}{cccc}
a_{0} & a_{1} & \cdots & a_{n-1} \\
a_{n-r} & a_{n-r+1} & \cdots & a_{n-r-1} \\
a_{n-2 r} & a_{n-2 r+1} & \cdots & a_{n-2 r-1} \\
\cdot & \cdot & & \cdot \\
\cdot & \cdot & & \cdot \\
\cdot & \cdot & & \cdot \\
a_{r} & a_{r+1} & \cdots & a_{r-1}
\end{array}\right]
$$

where $r$ is a nonnegative integer and each of the subscripts is understood to be reduced modulo $n$.

$C^{*}$ and $C^{+}$will denote respectively the conjugate transpose and MoorePenrose pseudoinverse of the $r$-circulant $C$. $(n, r)$ will denote the gcd of $n$ and $r$.

Definition 2. $P$ will be the 1-circulant with first row $e_{2}^{*}=[0,1,0, \cdots, 0]$. $Q_{r}$ will be the $r$-circulant with first row $e_{1}^{*}=[1,0, \cdots, 0]$.

Received by the editors June 15, 1971 and, in revised form, October 4, 1971.

AMS 1970 subject classifications. Primary 15A09.

Key nords and phrases. Pseudoinverse, r-circulant matrix.

(c) American Mathematical Society 1972 
Property 1 [3]. The Moore-Penrose pseudoinverse of a 1-circulant is a 1-circulant.

PROPERTY 2 [2]. The Moore-Penrose pseudoinverse of a nonsingular $r$-circulant in an $s$-circulant where $s$ satisfies $r s \equiv 1(\bmod n)$.

Property 3 [1]. $C$ is an $r$-circulant if and only if $P C=C P^{r}$.

Property 4 [1]. If $C$ and $D$ are $r$ - and $s$-circulants respectively, then $C D$ is an $r s$-circulant.

Property 5 [1]. Let $\omega_{1}, \omega_{2}, \cdots, \omega_{n}$ be the $n$th roots of unity and let $R_{i}^{*}=(1 / \sqrt{ } n)\left[1, \bar{\omega}_{i}, \bar{\omega}_{i}^{2}, \cdots, \bar{\omega}_{i}^{n-1}\right], i=1,2, \cdots, n$. Then $\left\{R_{i}: i=1,2, \cdots, n\right\}$ is an orthonormal set of eigenvectors for any $n \times n$ 1-circulant.

\section{Main results.}

THEOREM 1. $C$ is an $r$-circulant if and only if $\left(C^{+}\right)^{*}$ is an $r$-circulant.

Proof. By Property 3,C=P*CPr where $P$ is the unitary matrix described in Definition 2. Therefore

$$
\left(C^{+}\right)^{*}=\left(P^{r *} C^{+} P\right)^{*}=P^{*}\left(C^{+}\right) * P^{r} .
$$

Using Property 3 again, we see that $\left(C^{+}\right)^{*}$ is an $r$-circulant. The uniqueness of $C^{+}$gives the reverse implication.

We have said that if $C$ is a singular $r$-circulant, $C^{+}$may not be an $s$ circulant for any integer $s$. The $4 \times 4$ matrix $Q_{2}$ is an example. $Q_{2}^{+}=\frac{1}{2} Q_{2}^{*}$.

THEOREM 2. If $C$ is an $n \times n r_{1}$-circulant then $C^{+}$is an $s_{1}$-circulant if and only if there exists an integer $r$ such that $(n, r)=1$ and $C$ is also an $r$-circulant. In such a case there exists an integer such that $r s \equiv 1(\bmod n)$ and $C^{+}$is also an s-circulant.

In order to prove the "if" part of Theorem 2 we need two lemmas.

LEMMA 1. The $n \times n$ matrix $Q_{r}$ is unitary if and only if $(n, r)=1$.

Proof. From Definition 2 it is clear that

$$
Q_{r}=\left[\begin{array}{c}
e_{1}^{*} \\
e_{r+1}^{*} \\
\cdot \\
\cdot \\
\cdot \\
e_{(n-1) r+1}^{*}
\end{array}\right] .
$$

$Q_{r}$ will be singular if and only if there exist integers $i$ and $j$ satisfying $0 \leqq i<j<n$ such that $i r \equiv j r(\bmod n)$; that is, if and only if there exists an 
integer $b$ such that

$$
b n=i r-j r=(i-j) r .
$$

Since $|i-j|<n,(1)$ is valid if and only if $(n, r)>1$.

The following lemma is a result of Definitions 1 and 2 .

LEMMA 2. $C$ is an $r$-circulant with first row $\left[a_{0}, a_{1}, \cdots, a_{n-1}\right]$ if and only if $C=\sum_{i=0}^{n-1} a_{i} Q_{r} P^{i}$.

Proof of TheOrem 2 ("if" part only). If $C$ is also an $r$-circulant where $(n, r)=1$, then $C=\sum_{i=0}^{n-1} a_{i} Q_{r} P^{i}=Q_{r} C_{1}$ where $Q_{r}$ is unitary and $C_{1}$ is a 1-circulant with the same first row as $C$. By Properties 1,2 and $4, C^{+}=$ $C_{1}^{+} Q_{r}^{*}$ is an $s$-circulant where $r s \equiv 1(\bmod n)$.

Theorem 1 tells us that if we replace " $C^{+}$" with " $C$ *" in Theorem 2 we have a statement which is equivalent to Theorem 2 . Hence we can complete the proof of Theorem 2 by proving the "only if" part for $C^{*}$.

LEMMA 3. If $C$ is an $r$-circulant with first row $\left[a_{0}, a_{1}, \cdots, a_{n-1}\right]$ then $C^{*}$ is an s-circulant if and only if $a_{i r s}=a_{i}, i=0,1, \cdots, n-1$, where the subscripts are understood to be reduced modulo $n$.

Proof. Referring to Definition 1, the first column of $C^{*}$ can be written as both

$$
\left[\begin{array}{c}
\bar{a}_{0} \\
\bar{a}_{1} \\
\bar{a}_{2} \\
\cdot \\
\cdot \\
\cdot \\
\bar{a}_{n-1}
\end{array}\right] \text { and }\left[\begin{array}{c}
\bar{a}_{0} \\
\bar{a}_{r s} \\
\bar{a}_{2 r s} \\
\cdot \\
\cdot \\
\cdot \\
\bar{a}_{(n-1) r s}
\end{array}\right] \text {. }
$$

The lemma follows from equating like elements and observing that $C^{*}$ is completely described by specifying $r$ and the first column of $C^{*}$.

Proof of Theorem 2 ("only if" part). Suppose $C$ and $C^{*}$ are $n_{1} \times n_{1}$ $r_{1}$ - and $s_{1}$-circulants, respectively, such that $\left(r_{1}, n_{1}\right)>1$ and $\left(s_{1}, n_{1}\right)>1$. If $r_{1}=0$ or $s_{1}=0$ every element of $C$ must be the same and $C$ is an $r$ circulant for any $r$ such that $0 \leqq r<n$. If $r_{1} \neq 0$, define $n_{2}=n_{1} /\left(r_{1} s_{1}, n_{1}\right)$ and $k_{1}=n_{1} / n_{2}$. By Lemma 3, $a_{i}=a_{n_{2}+i}=a_{2 n_{2}+i}=\cdots=a_{\left(k_{1}-1\right) n_{2}+i}, i=0,1, \cdots$, $n_{1}-1$. Thus $C$ must be a composite of $k_{1}^{2}$ identical $n_{2} \times n_{2}$ matrices. Define $r_{2}$ to be the modulo $n_{2}$ residue of $r_{1}$. Each of the $n_{2} \times n_{2}$ matrices is an $r_{2^{-}}$ circulant and $C$ is an $h$-circulant for all $h \in\left\{r_{2}+j n_{2}: j=0,1,2, \cdots, k_{1}-1\right\}=$ $H$. If $\left(r_{2}, n_{2}\right)=1$ the collection $\left\{r_{2}+j n_{2}: j=0, \pm 1, \pm 2, \cdots\right\}=J$ contains infinitely many primes larger than $n_{1}$. Each element in $J$ is congruent 
modulo $n_{1}$ to some element in $H$. But a prime larger than $n_{1}$ is congruent modulo $n_{1}$ to an element in the reduced residue system of $n_{1}$. Thus there exists an $r \in H$ such that $\left(r, n_{1}\right)=1$ and $C$ is an $r$-circulant. If $r_{2}=0$ the situation is the same as when $r_{1}=0$.

If $\left(r_{2}, n_{2}\right)>1$, define $s_{2}$ to be the modulo $n_{2}$ residue of $s_{1}$ and repeat the above with $n_{2}, n_{3}, r_{2}, s_{2}$ and $k_{2}$. If $\left(r_{3}, n_{3}\right)>1$ continue to repeat the process until $\left(r_{m}, n_{m}\right)=1$ or $r_{m}=0$ for some $m$. The latter case yields the same situation as $r_{1}=0$ If $r_{i} \neq 0$ for any $i$ we can guarantee that an $m$ does exist such that $\left(r_{m}, n_{m}\right)=1$ since $n_{i}$ divides $n_{i-1}, i=2,3, \cdots, m$. Now $C$ is an $h$-circulant for all $h \in\left\{r_{m}+j n_{m}: j=0,1, \cdots, k_{m-1}-1\right\}=H_{m}$. As before, we can find an $r \in H_{m}$ such that $\left(r, n_{1}\right)=1$.

Corollary 1. If $C$ and $D$ are $n \times n$-circulants then $C D^{*}$ is a 1circulant. If $(n, r)=1$ then $C^{*} D$ is a 1-circulant.

Proof. $C D^{*}$ is a 1-circulant since $C D^{*}=P^{*} C P^{r} P^{r *} D^{*} P=P^{*} C D^{*} P$.

We can now conclude that if $C$ is an $r$-circulant, then $C C^{+}$must be a 1-circulant, but $C^{+} C$ may not be unless $(n, r)=1$. The $4 \times 4$ matrix $Q_{2}$ illustrates this point.

Calculation of $C^{+}$is simple. Let $R$ be the matrix whose columns are the $R_{i}$ defined in Property 5. $R^{*} C C^{*} R=D$ is a diagonal matrix and $C^{+}=$ $C^{*}\left(C C^{*}\right)^{+}=C^{*} R D^{+} R^{*}$. If there exists an $r$ such that $C$ is an $r$-circulant and $(n, r)=1$ the following formula is also valid and requires one less matrix multiplication:

$$
C^{+}=C_{1}^{+} Q_{r}^{*}=R D_{1}^{+} R^{*} Q_{r}^{*}
$$

where $C_{1}$ is defined as in the proof of Theorem 2 and $R^{*} C_{1} R=D_{1}$.

\section{BIBLIOGRAPHY}

1. C. M. Ablow and J. L. Brenner, Roots and cannonical forms for circulant matrices, Trans. Amer. Math. Soc. 107 (1963), 360-376. MR 27 \#5775.

2. S. Charmonman and R. S. Julius, Explicit inverses and condition numbers of certain circulants, Math. Comp. 22 (1968), 428-430. MR 37 \#2418.

3. G. H. Worm, Explicit solutions for quadratic minimization problems, Doctoral Dissertation, University of Tennessee, Knoxville, Tennessee, 1971.

4. I. Niven and H. S. Zuckerman, An introduction to the theory of numbers, Wiley, New York, 1960. MR 22 \#5605.

Department of Mathematics, United States Air force Academy, Colorado 80840

Department of Mathematics, Texas Tech University, Lubbock, Texas 79409 